\title{
LEIBNIZ Y EL LABERINTO DE LA NADA: LA ONTOLOGÍA EN MANOS DE UN ESCOLÁSTICO FURTIVO
}

\author{
Dr. (C) Camilo Silva ${ }^{1}$ \\ Escuela Normal Superior de París, Francia
}

\begin{abstract}
Resumen: En sus escritos filosóficos estrictamente privados, Leibniz intenta definir sistemáticamente el concepto 'nada'. Sin haber despertado un interés particular en los estudiosos de Leibniz, el valor de tales escritos recae en el carácter aporético que revisten y que exhortan a Leibniz a buscar una salida al laberinto ontológico al cual ellos conducen: el laberinto de la nada. El propósito del presente estudio consiste en revelar las dificultades envueltas en -lo que llamamos- la doctrina leibniziana de la nada, destacando la importancia que ésta tiene a la luz de la ontología general de Leibniz. Por medio de un examen analítico de diversos escritos, intentaremos mostrar de qué manera tales dificultades conducen a Leibniz a forjar una teoría concebibilista de la nada, convirtiéndose furtivamente en lo que él rechaza: un escolástico.
\end{abstract}

Descriptores: Concebibilidad $\cdot$ Eliminativismo $\cdot$ Nominalismo $\cdot$ No-ser $\cdot$ Realismo.

Abstract: In his strictly private philosophical papers, Leibniz systematically tries to define the concept 'nothingness'. Although Leibniz scholars have not paid special attention to them, the value of these pieces rests on both the aporetic character they have and the need Leibniz had to find a way out from the ontological labyrinth where they lead: the labyrinth of nothingness. The task of this research is to reveal the difficulties involved in -what we call itthe Leibnizian doctrine of nothingness, pointing out its importance in the light of Leibniz's general ontology. Through an analytic survey of several Leibniz's personal manuscripts, we will try to expose the way in which the difficulties Leibniz threats lead him to admit that nothingness is conceivable. Furtively Leibniz becomes thus in what he rejects: A Scholastic.

Keywords: Conceivability $\cdot$ Eliminativism $\cdot$ Nominalism $\cdot$ Not-being $\cdot$ Realism

Enviado: 23/04/2020 Aceptado: 11/05/2020

\footnotetext{
${ }^{1}$ Este estudio forma parte de mi investigación doctoral realizada actualmente en la Escuela Normal Superior de París y que ha sido financiada en un primer período por el programa CONICYT-Becas Chile (2015-2019) y, en un segundo período, por un contrato doctoral concursable otorgado por la misma institución en que llevo a cabo mis estudios (2019-2020). Una versión preliminar de este estudio fue presentada oralmente en el Premier colloque francophone de jeunes chercheurs leibniziens que tuvo lugar en la Escuela Normal Superior de París (2018). Agradezco las pertinentes, valiosas y agudas observaciones que, en dicha presentación, los participantes me hicieron ver, en particular las de JeanPascal Anfray, Ohad Nachtomy y Jean-Baptiste Rauzy.
} 


\section{INTRODUCCIÓN}

\section{Describir a Leibniz (1646-1716) como autor de una doctrina de la nada} puede parecer, a primera vista, más que un despropósito o un error interpretativo, un pretencioso sinsentido. Opositor de las corrientes escolásticas que reducen la metafísica a un mero rigorismo verbal, Leibniz adhiere, ya desde su juventud, a la escuela nominalista, que él juzga como "la más profunda entre todas las escuelas de las corrientes escolásticas". En este sentido, pese a que, a lo largo del tiempo, Leibniz atenúa los alcances de su adhesión al nominalismo, al incorporar conceptos provenientes de la escolástica para justificar ciertas doctrinas metafísicas, ${ }^{3}$ pareciera que él no abandona jamás el nominalismo como un precepto metodológico de su filosofía. ${ }^{4}$ Sin embargo, entre los innumerables manuscritos filosóficos de Leibniz, existe un conjunto considerable de documentos en los que él intenta sistemáticamente definir el concepto 'nada' (Nihil). Sin respaldar la contestable interpretación de Russell, quien acusa a Leibniz de ser autor de dos filosofías, ${ }^{5}$ es indudable que, en sus documentos de uso privado, éste aborda problemas - por medio de un singular aparato crítico- que no son tratados en sus escritos divulgados o publicados: entre ellos, figura el problema de la nada. En tales manuscritos personales, Leibniz hace de dicho concepto un objeto de la metafísica o, más precisamente, de la ontología, siendo así conducido al 'laberinto de la nada'. Los manuscritos en los que Leibniz sistematiza su doctrina de la nada constituyen una prueba indesmentible de una

\footnotetext{
${ }^{2}$ Dissertatio praeliminaris Marii Nizolii de veris principiis et vera ratione philosophandi libri IV (1670), Sämtliche Schriften und Briefe, (1923-.), Berlin-Brandenburgische Akademie der Wissenschaften et al. (ed.). Darmstadt, Leipzig, Berlín, Akademie-Verlag, serie VI, tomo 2, p. 427. En lo sucesivo, siguiendo el empleo estándar para las referencias de las obras de Leibniz, para la edición recientemente mencionada, utilizaremos "A", seguido del número de serie, tomo y página. Asimismo, utilizaremos "G" para G.W. Leibniz Textes inédits (1998), G. Grua (ed.), 2 vols., Paris, Presses Universitaires de France, y "GP" Philosophischen Schriften (1875-1890), C. I. Gerhardt (ed.), 7 volúmenes, Berlín (reimpresión Hildesheim 1960-1961), seguidos del volumen y página. Las traducciones de las citas son del autor de este artículo, a menos que se indique expresamente lo contrario.
}

${ }^{3}$ Además de la temprana rehabilitación de las formas substanciales (cf. A Johann Friedrich, 1679, A II, 1, 754), Leibniz, en su filosofía tardía, invoca el concepto de Vinculum substantiale para explicar la relación que mantienen las mónadas subordinadas con la mónada dominante (cf. A Des Bosses, 5 de febrero de 1712, GP II, 435). Sobre la rehabilitación leibniziana de las formas substanciales, cf. M. Fichant (1998), en particular capítulo VII. Sobre el concepto de vinculo substancial en Leibniz, cf. B. Look (1999).

${ }^{4}$ En el marco de la discusión de la realidad de las entidades abstractas -cuyo uso Leibniz proscribe-, él mismo se declara nominalista: "Hasta ahora, yo no veo otra manera de evitar estas dificultades que considerando los abstractos no como cosas, sino como abreviaciones [compendia loquendi] [...] Y en esta medida, yo soy un nominalista, al menos de manera provisoria" (De realitate accidentium (1688 (?)), A VI, 4-A, 996). La cuestión del alcance del nominalismo de Leibniz ha sido ampliamente discutida. En este contexto, nos limitamos a indicar algunos estudios de referencia: L. Couturat (1961), pp. 457-472; B. Mates (1979), (1980) y (1986) pp. 170-188; M. Mugnai (1992), pp. 22-27; y D. Rutherford (1995), pp. 115-119.

5 "una (...) optimista, ortodoxa, fantástica y superficial [y] otra, profunda, coherente, en gran medida spinozista y asombrosamente lógica" (B. Russell (2005), p. 531). 
ruptura con el nominalismo. Al entrar en este laberinto, Leibniz se convierte furtivamente en lo que rechaza: un escolástico.

El propósito del presente estudio consiste en poner a la luz la doctrina leibniziana de la nada, describiendo analíticamente las tesis más substanciales que la definen, así como las dificultades que de ella se desprenden. Para tales efectos, hemos dividido este estudio en cinco secciones. En la primera, concentrándonos en la correspondencia con Thomasius y en la Dissertatio praeliminaris de De veris principiis et vera ratione philosophandi de Mario Nizolius, explicaremos el juvenil rechazo del escolasticismo de Leibniz y su adhesión al nominalismo. En la segunda sección, evocaremos la definición que, sobre la base del cálculo lógico, Leibniz proporciona del concepto 'nada'. En la tercera sección, expondremos la tensión entre dos criterios con los que Leibniz caracteriza dicho concepto: mientras que, a partir de un enfoque eliminativista, el concepto 'nada' es reducido al de 'imposible', sobre la base de un criterio epistémico, el mismo concepto parece ser tenido por concebible. En virtud de los resultados del análisis de la tercera sección, en la cuarta analizaremos el problema de la "realidad" de la nada, tesis a la que Leibniz parece suscribir en algunos textos. Finalmente, en la quinta sección, a partir de la distinción escolástica invocada por Leibniz entre no-ser como negación o privación (negatio - privatio) y no-ser como no-ser de razón (non-ens rationis), intentaremos justificar una interpretación concebibilista del concepto 'nada' en su ontología, en detrimento de las otras dos interpretaciones posibles: eliminativista y realista.

\section{Anti-esColasticismo y NOMINALISMO EN LOS ESCRITOS JUVENILES DE LEIBNIZ}

Tras abordar el clásico problema del principio de individuación en la homónima disertación de $1663,{ }^{6}$ el joven Leibniz reorienta sus intereses filosóficos: aunque las discusiones metafísicas seguirán teniendo una relevancia innegable, éstas, en el mismo decenio, aparecen en un contexto teórico diferente. Seducido por los filósofos modernos, ${ }^{7}$ Leibniz hace suyos los problemas y propósitos expuestos por aquéllos. En este cuadro, la filosofía natural cobra un significativo realce en su pensamiento, al punto que, en cierto sentido, ella se convierte en uno de los núcleos teóricos en torno al cual gira una gran parte de sus preocupaciones filosóficas. ${ }^{8}$

\footnotetext{
${ }^{6}$ Disputatio Metaphysica de principio individui, A VI, 1, 3-19.
}

${ }^{7}$ Dentro de los cuales Leibniz incluye -de manera relativamente invariable- a Galileo, Bacon, Gassendi, Hobbes, Descartes y Digby. Cf. A Thomasius, 6 de octubre de 1668, A II, 1, 18; Confessio naturae contra atheistas (1668-1669 (?)), A VI, 1, 489-490, y A Spener, 10/20 de febrero de 1670, A II, 1, 56.

${ }^{8}$ Para ser más precisos, aunque la filosofía natural constituye una parte central de las investigaciones de Leibniz en aquella época, su desarrollo no es autónomo, ya que se encuentra supeditada a los principios teológicos con los que ella debe conciliarse. Para una descripción acabada de la articulación de estos diversos intereses -de los que forma parte, además, un conjunto masivo de manuscritos de derecho natural-, cf. M. R. Antognazza (2009), en particular, primera parte. 
Con el objeto de exponer el modo en que se articulan el anti-escolasticismo y el nominalismo en el joven Leibniz, abordaremos, primero, algunos escritos de la correspondencia que, entre 1663 y 1672, éste sostuvo con su maestro, Jacobo Thomasius, y luego la Dissertatio praeliminaris que precede la reedición de 1670 de De veris principiis et vera ratione philosophandi de Mario Nizolius.

\subsection{LA CORRESPONDENCIA CON THOMASIUS}

Los primeros trazos de la filosofía natural de Leibniz aparecen en la correspondencia que él sostuvo con su maestro, Jacobo Thomasius (1622-1684). ${ }^{9}$ Siguiendo a este último, Leibniz reconoce el valor de la filosofía natural (física) de Aristóteles. Sin embargo, él piensa, además, que la filosofía de Aristóteles puede y debe conciliarse con la filosofía de los modernos ${ }^{10}$. Definida en tales términos, esta conciliación, destinada a establecer la reforma de la filosofía (Philosophia Reformata), entraña una tensión, dado el rechazo de la tradición escolástica expresada por parte de los filósofos modernos. La estrategia de Leibniz consiste así, por una parte, en invalidar las explicaciones escolásticas en beneficio de las explicaciones mecanicistas de los modernos y, por otra, en disociar la filosofía de Aristóteles de los escolásticos, mostrando que las interpretaciones de éstos no representan fielmente el pensamiento del estagirita. ${ }^{11}$

En su carta del 20/30 de abril de 1669, Leibniz enuncia las condiciones metodológicas de la ciencia, subrayando, al mismo tiempo, las deficiencias de la tradición escolástica al no regirse por ellas. Como él explica,

las garantías de esta ciencia deben establecerse a partir de la sensación, la experiencia y las matemáticas, instrumentos de los cuales los escolásticos (...) han estado casi totalmente desprovistos. Así, es muy probable que en Física se hayan mal encaminado. ${ }^{12}$

Leibniz denuncia que es en virtud de la inadecuación de los recursos metodológicos de la tradición escolástica que sus explicaciones no dan cuenta satisfactoriamente de los fenómenos de la naturaleza. En contraste con ella, los modernos sí proveen

\footnotetext{
${ }_{9}^{9}$ Para un análisis de la correspondencia entre Leibniz y Thomasius, cf. C. Mercer (2004).

${ }^{10}$ Cf. A Thomasius, 6 de octubre de 1668, A II, 1, 18.

11 "Las obscuridades de Aristóteles son el resultado del humo escolástico" ( $A$ Thomasius, 6 de octubre de 1668, A II, 1, 18): "casi en ninguna parte él [Aristóteles] dice lo que los escolásticos le han atribuido" (Hypothesis physica nova -también llamada Theoria motus concreti- (1670-1671 (?)), §56, A VI, $2,247)$. Consignemos -dicho sea de paso- que esta estrategia de reivindicación de Aristóteles, distinguiendo su filosofía de la de los escolásticos, se remonta ya a la época renacentista. Sobre este punto, cf. C. Schmitt (1971 y 1983).
}

${ }^{12}$ A Thomasius, 20/30 de abril de 1669, A II, 1, 26. 
explicaciones científicas satisfactorias, ya que sus hipótesis sí integran tales requisitos metodológicos. En efecto,

aunque la explicación de los escolásticos y la de los modernos sean una y otra posibles, entre dos hipótesis posibles, es necesario elegir la más clara e inteligible. Y tales son las cualidades que posee indudablemente la hipótesis de los modernos, la que no inventa ninguna entidad incorporal en los cuerpos y, por el contrario, no asume más que la magnitud, la figura y el movimiento. ${ }^{13}$

Dado que las hipótesis de los modernos, al hacer uso de conceptos matemáticos y empíricos tales como los de magnitud, figura y movimiento, resultan más claras e inteligibles que las de los escolásticos, las explicaciones de éstos deben ser descartadas en beneficio de las de aquéllos: "las hipótesis de la filosofía reformada -observa Leibniz- tienen, por sobre las de los escolásticos, la ventaja de no ser superfluas, sino, por el contrario, esclarecedoras". ${ }^{14}$

Ahora bien, si, para emplazar su estrategia de conciliación entre Aristóteles y los filósofos modernos, Leibniz intenta establecer una equivalencia semántica respectiva entre los conceptos aristotélicos de materia, forma y cambio con los de masa, figura y movimiento de los modernos, ${ }^{15}$ para distinguir la filosofía de Aristóteles de la de los escolásticos, Leibniz levanta diversas críticas del concepto de forma substancial tal como es usado por éstos.

Como sabemos, antes de la aparición de la revolucionaria filosofía de Descartes, el concepto de forma substancial constituía uno de los recursos teóricos predilectos de la tradición escolástica para explicar los fenómenos naturales. En el contexto de la correspondencia con Thomasius, Leibniz elabora, al menos, tres argumentos para desacreditar el uso escolástico de las formas substanciales: de éstos, dejaremos al margen dos, y nos concentraremos en el más relevante para nuestra discusión. ${ }^{16}$

En la misma carta del 20/30 de abril de 1669, Leibniz acusa la vacuidad semántica y esterilidad explicativa de las formas substanciales debido a que son inimaginables. Según él,

${ }^{13}$ Ibid., A II, 1, 26. Cf. también Confessio naturae contra atheistas, A VI, 1, 489-490 e Hypothesis physica nova, §57, A VI, 2, 247-249.

${ }^{14}$ A Thomasius, ibid., A II, 1, 35.

${ }^{15}$ Cf. ibid., A II, 1, 25-29. Para un análisis en detalle de los términos en los que Leibniz intenta concretamente establecer esta conciliación, cf. C. Mercer (1990) y D. Garber (2009), en particular pp. 1-10.

${ }^{16}$ El primero de tales argumentos consiste en que la atribución de formas substanciales a los cuerpos vuelve superflua la existencia de un primer motor del cual-Leibniz adopta, en esta época, una posición ocasionalista- todo depende, contradiciendo así la filosofía misma de Aristóteles (cf. A Thomasius, 6 de octubre de 1668, A II, 1, 19). El segundo argumento apunta a disociar el significado que Aristóteles atribuye a las formas substanciales del sentido en que éstas son entendidas por los escolásticos (cf. $A$ Thomasius, 20/30 de abril de 1669, A II, 1, 32). 
El espíritu no puede imaginarse más que el espíritu mismo (cuando se piensa a sí mismo, ciertamente), el espacio, la materia y lo que resulta de sus relaciones mutuas. Todo lo que uno podría añadir a ello no son más que palabras: uno puede pronunciarlas y combinarlas entre sí de manera variada, pero no explicarlas, ni comprenderlas. ¿Quién puede, en efecto, imaginarse una entidad que no participaría ni de la extensión, ni del pensamiento? ¿De qué sirve entonces suponer almas incorporales en los animales y en las plantas, [o] formas substanciales en los elementos y en los metales, carentes de extensión? ${ }^{17}$

Supuesta su superfluidad, las formas substanciales -tal como son caracterizadas por los escolásticos- no constituyen, para Leibniz, un principio de explicación razonable: al no poder ser imaginadas, explicadas o comprendidas, ellas no dan razón de los fenómenos naturales.

En el fondo, la premisa que subyace a la posición refractaria de Leibniz frente a las formas substanciales es que éstas carecen de significado. Así, es en el contexto de la discusión sobre el valor de las hipótesis para explicar los fenómenos naturales que Leibniz adhiere subrepticiamente al nominalismo: si las hipótesis escolásticas deben ser descartadas en beneficio de las de los modernos es porque violan el principio de economía. De este modo, la claridad e inteligibilidad de las explicaciones son entendidas por Leibniz como un criterio epistemológico que valida las explicaciones debido a su carácter económico: simplex sigillum veri. Y es en este punto -piensa Leibniz- en donde la filosofía de Aristóteles y la de los modernos convergen, separándose de los escolásticos, cuyas hipótesis poseen un menor poder explicativo. Síntesis de la posición de Leibniz -apologética, por un lado, en relación con los modernos y Aristóteles, y crítica, por otro, en relación con la escolástica- a la luz de este nominalismo gestacional, es el siguiente pasaje:

Las hipótesis de los modernos son las mejores, puesto que ellos no utilizan más que eso [el espíritu, la materia, el espacio y el movimiento] para dar cuenta de los fenómenos. La deficiencia de una hipótesis es, en efecto, asumir aquello que no es necesario. Todas las cosas (...) pueden explicarse a partir sólo de tales datos, como sugiere la lectura de los filósofos modernos (...) mostrando la posibilidad de una conciliación con Aristóteles (...). Las mejores hipótesis son aquéllas que resultan más claras. ${ }^{18}$

\subsection{La Dissertatio PRAELIMinaris Del libro de Nizolius}

La Dissertatio praeliminaris corresponde a un texto en el que Leibniz introduce críticamente la doctrina que el humanista italiano Mario Nizolius (14981576) expone en su libro De veris principiis et vera ratione philosophandi que,

\footnotetext{
${ }^{17}$ A Thomasius, 20/30 abril 1669, A II, 1, 34-35.

${ }^{18}$ Ibid., A II, 1, 34.
} 
publicado originalmente en 1553 , es reeditado en $1670 .{ }^{19}$ La Dissertatio praeliminaris es, por lo tanto, redactada -o, al menos, preparada- en el mismo período en el que tienen lugar las piezas epistolares con Thomasius que hemos evocado. Dada la sincronía en el desarrollo de estos dos focos teóricos, existe un entrelazamiento de ideas que, en su conjunto, permiten restituir la filosofía del joven Leibniz. Pese a esto, tanto los problemas como el tratamiento que de ellos Leibniz ejerce en la Dissertatio, preservan una singularidad irreductible. Muestra del carácter parcial de esta superposición teórica es el siguiente pasaje de la Dissertatio, en el que Leibniz alude nuevamente al principio de economía:

Una hipótesis es mejor en la medida en que es más simple y (...) cuando se trata de encontrar la causa de los fenómenos, procede de mejor forma aquél que supone menos cosas de manera gratuita. Así, pues, aquél que procede de otra forma, imputa a la naturaleza o al Autor de ésta, un derroche irrazonable (...) De esta regla, los nominalistas han deducido que todo en la naturaleza podía explicarse incluso aunque no hubiese universales ni formalidades reales: no hay nada más cierto. ${ }^{20}$

$\mathrm{Si}$, tal como en la correspondencia con Thomasius, Leibniz destaca la simplicidad de las hipótesis como criterio para determinar su valor epistemológico, la apología del principio de economía en la Dissertatio aparece, derechamente, delante del telón que le da vida: el nominalismo. Es éste -más que la filosofía natural- el eje que, en gran medida, articula el desarrollo argumentativo de Leibniz en la Dissertatio praeliminaris. $^{21}$

La preocupación central de Leibniz en la Dissertatio es analizar las cualidades que hacen posible el discurso filosófico, y que para él son la claridad, la verdad y la certeza. Al definir esta última como la claridad de la verdad, Leibniz concibe la claridad como la condición más elemental del discurso filosófico. ${ }^{22}$ Leibniz sostiene que, en su nivel fundamental, la claridad es una cualidad que afecta a las palabras mismas: "la verdad de una proposición no puede ser conocida si el significado de las

\footnotetext{
${ }^{19}$ En este sentido -dicho sea de paso- la Dissertatio praeliminaris es un comentario crítico de la obra de Nizolius, en la que Leibniz presenta sus propias credenciales filosóficas: "Hemos editado -escribe Leibniz- [el libro de] Nizolius con el fin de contribuir, aunque sea por la obra de otro, en algo a la instauración de una filosofía más sólida (...) De este modo, esperamos que la lectura de este comentario de [la obra de] Nizolius sea fructífera para la filosofía" (A VI, 2, 420-421). Para una comprensión de la recepción y crítica de Leibniz de la filosofía de Nizolius, cf. Angelelli (1965 y 2004) y J.-B. Rauzy (2001), pp. 184-200.

${ }^{20}$ Dissertatio praeliminaris, A VI, 2, 428.

${ }^{21}$ Leibniz expone su filosofía natural en dos textos que datan de la misma época: Hypothesis physica nova (1670-1671 (?)), A VI, 2, 219-257 y Theoria motus abstracti (1670-1671 (?)), A VI, 2, 258-276.

22 "La certeza se revelará como no siendo sino la claridad de la verdad (...) la claridad y la verdad se derivan de la noción de 'certeza' tomada en sí misma" (Dissertatio praeliminaris, A VI, 2, 409).
} 
palabras no es conocido, es decir (...) si la proposición no es clara". ${ }^{23}$ Así, la labor del filósofo consiste en "hablar de la claridad de las palabras mismas". ${ }^{24}$ Éste es el terreno sobre el cual Leibniz, al adoptar el nominalismo como método del discurso filosófico, puede profundizar su crítica contra la escolástica.

La estrategia de Leibniz en la Dissertatio es acusar la vacuidad semántica de los conceptos técnicos: "los términos técnicos -sostiene- deben ser evitados como la peste". ${ }^{25}$ Dado esto, Leibniz exhorta a regirse por el uso de -lo que él llamaconceptos populares u ordinarios. Tal es la contraposición que Leibniz explica en el siguiente pasaje:

Ya que es una constante que no hay cosas que no puedan expresarse mediante [el uso de] términos populares [y] que es también una constante que un discurso es más claro en la medida en que sus términos son más populares (...) es manifiesto que la regla o medida para el empleo de los términos debe ser la popularidad más económica o la economía más popular. Por lo tanto, allí donde hay términos populares y económicos en abundancia, es necesario abstenerse de los términos técnicos. ${ }^{26}$

Supuesto este cuadro general, la adopción del nominalismo por parte de Leibniz se hace más patente cuando el principio de economía es directamente aplicado al uso de los conceptos filosóficos. Desde una perspectiva ockamista, Leibniz juzga que sólo "aquello que es concreto es verdaderamente real", ${ }^{27}$ de manera que "las abstracciones no son reales, sino modos de las cosas reales". ${ }^{28}$ Por lo tanto, todo lenguaje que, apelando a términos abstractos, transgreda el uso de conceptos corrientes, debe ser proscrito, y ciertamente, para Leibniz, tal es el caso del lenguaje escolástico: si éste es ya objeto de reproche por parte de Leibniz en sus cartas a Thomasius, ${ }^{29}$ esta crítica se agudiza en la Dissertatio.

\footnotetext{
${ }^{23}$ Ibid.

${ }^{24}$ Ibid.

${ }^{25}$ Ibid., A VI, 2, 411.

${ }^{26}$ Ibid., A VI, 2, 415. La misma apología del uso del lenguaje corriente aparece en una carta que Leibniz dirige a Thomasius en septiembre de 1669: "este lenguaje [racional] no es en lo absoluto técnico, sino que está inspirado en el lenguaje corriente (...). Gracias a él, es posible enunciar adecuadamente, de manera pura y precisa, todas las sutilizas que uno quiera (...). Si algo puede ser expresado con la misma facilidad mediante términos populares, es necesario abstenerse de las elucubraciones técnicas que han sido fabricadas para obscurecer [aquello que se dice de] algo" (A Thomasius, septiembre de 1669, A II, 1, 42).

${ }^{27}$ Dissertatio praeliminaris, A VI, 2, 417.

${ }^{28}$ Ibid. Y -continúa Leibniz- "la mayor parte de los modos no son más que relaciones de la realidad con el entendimiento (...)" (Ibid.).

29 "Los escolásticos se han expresado, no sólo barbáricamente, sino que también con muy poca precisión" (A Thomasius, septiembre 1669, A II, 1, 42).
} 
Para Leibniz, los escolásticos en vez de preocuparse por el cuidado y correcto uso del lenguaje, no han hecho más que "acumular referencias teóricas, inventar innumerables preguntas ociosas, dividir un sólo argumento en varios, cambiar de método e imaginar y volver a imaginar términos nuevos". ${ }^{30}$ En este sentido, junto con denunciar algunos conceptos como vacuos y, por lo tanto, inaceptables, ${ }^{31}$ Leibniz ridiculiza el abstraccionismo escolástico, poniendo a la luz las contradicciones que éste engendra. Como él explica:

$\mathrm{Si}$, en efecto, la entidad es un ser, si la realidad es una cosa, si la coseidad [aliquidditas] es una cosa, la misma cosa será la forma de sí misma o una parte de su propio concepto, lo que implica contradicción. Por consiguiente, si alguien debe, algún día, componer los Elementos de la Filosofía de manera perfecta, es necesario que él descarte casi por completo las abstracciones. ${ }^{32}$

Ahora bien, además de la claridad de las palabras, la certeza no puede obtenerse sino a condición de disponer de un método que regule las relaciones que los conceptos mantienen entre sí. Leibniz ve, ciertamente, en la lógica el instrumento adecuado para que el discurso filosófico, sintácticamente ordenado, pueda proporcionar certezas, pues la verdadera lógica

contiene también (...) los principios y el método para elaborar la verdadera filosofía. En efecto, ella enseña las reglas generales gracias a las cuales es posible discernir lo verdadero de lo falso y demostrar todas las conclusiones recurriendo solamente a las definiciones y experiencias (...) Estos principios (...) formarán al filósofo y serán los principios de la verdadera forma de hacer filosofía. ${ }^{33}$

Pese a que la lógica sirva de mecanismo para establecer el discurso filosófico, es ella misma la que, irónicamente, guiará a Leibniz a forjar una doctrina de la nada. En otros términos, es el uso del cálculo lógico aplicado al concepto 'nada' lo que lo conducirá a abrir la puerta de entrada de este laberinto ontológico, abandonando su compromiso con el nominalismo.

\footnotetext{
${ }^{30}$ Dissertatio praeliminaris, A VI, 2, 427.

${ }^{31}$ Leibniz considera los conceptos 'depender', 'inherir', 'emanar' e 'influir' como metafóricos (cf. Ibid., A VI, 2, 418). Del mismo modo, él observa que el verbo 'ser' formado a partir del substantivo no es indispensable para la filosofía (cf. Ibid., A VI, 2, 415). Asimismo, Leibniz critica -ya en un sentido más amplio- la definición de la metafísica como 'ciencia del ser': "es vano decir con los escolásticos que el sujeto de la Metafísica es el ser en tanto que ser (...)” (Notas al Liber primus de Nizolius, A VI, 2, 463, nota 59).

${ }^{32}$ Dissertatio praeliminaris, A VI, 2, 417.

${ }^{33}$ Ibid., A VI, 2, 408.
} 


\section{LA DEFINICIÓN DEL CONCEPTO 'NADA'}

Como hemos anticipado, es en la lógica en donde se anida y desarrolla la doctrina leibniziana de la nada: al extender su uso al concepto 'nada', Leibniz entra en un laberinto ontológico del que jamás saldrá. ${ }^{34}$

En una de sus primeras tablas definicionales, ${ }^{35}$ Leibniz caracteriza el concepto 'nada' (Nihil) como "aquello que puede ser nombrado, [pero] que no puede ser pensado: es el nombre sin una cosa [o] un sonido sin significado en el pensamiento". ${ }^{36}$ Así pues, en perfecto acuerdo con su adhesión al nominalismo y su anti-escolasticismo, Leibniz caracteriza el concepto en cuestión como un término desprovisto de significado, esto es, como un flatus vocis. Sin embargo, pese a que, en escritos posteriores, Leibniz hará uso de esta caracterización económica y puramente negativa de dicho concepto, ${ }^{37}$ ella no será la más recurrente: en su remplazo, Leibniz privilegiará, pues, una definición del concepto 'nada'. Éste es el paso que conducirá a Leibniz al "laberinto de la nada": alejándose del nominalismo, él se convierte en un escolástico.

La primera definición del concepto 'nada' -que, de ahora en adelante, abreviaremos por comodidad 'DCN'- aparece en la misma tabla definicional, a modo de glosa que Leibniz añade posteriormente en el escrito mismo. Apelando al cálculo lógico, Leibniz establece la DCN en los siguientes términos:

Si 'N' no es 'A', 'N' no es 'B', 'N' no es 'C'y así sucesivamente, entonces se dice que 'N' no es nada [Nihil]: esto corresponde a lo que se dice vulgarmente [a saber] que el no-ser no tiene atributos [Non Entis nulle esse attributa]. ${ }^{38}$

\footnotetext{
${ }^{34}$ El hecho de que en el $§ 7$ de los Principes de la nature et de la grâce fondés en raison (1714) Leibniz intente validar la célebre y controversial pregunta ‘¿Por qué hay algo más bien que nada?' sobre la premisa de que "la nada es más simple y fácil que algo", parece, en efecto, sugerir una implícita “ontologización" de la nada (cf. GP, VI, 602). Pese a que, ciertamente, el significado de 'nada' guarde una estrecha relación con la pregunta recientemente enunciada, dada la naturaleza de este estudio, no podremos extendernos sobre este punto, limitándonos solamente a referirnos ocasionalmente a dicho vínculo de manera marginal, sugestiva y conjetural. Cf. F. Wolff (2007).

${ }^{35}$ La elaboración de tablas definicionales es una de las técnicas de trabajo predilectas que Leibniz emplea, casi incesantemente, a lo largo de su vida: "He fabricado muchas definiciones que deseo, algún día, poder ordenar [ranger]" (A Bourguet, 22 de marzo 1714, GP III, 569). Sobre este punto, cf. L. Couturat (1961), pp. 168-172.

${ }^{36}$ Vorarbeiten zur characteristica universalis (1671-1672 (?)), A VI, 2, 487.

${ }^{37}$ Cf. e.g. Definitiones notionum ex Wilkinso. Genera et Species, causa et effectus (1677-1686 (?)), A VI, 4-A, 27 y Definitiones : aliquid, nihil, opposita, possibile (1688-1689 (?)), A VI, 4-A, 937.

${ }^{38}$ Vorarbeiten zur characteristica universalis, A VI, 2, 487.
} 
Al margen de la inadecuación que, en el fondo, encierra la DCN formulada en tales términos, ${ }^{39}$ Leibniz la invocará regular y casi invariablemente en varios de sus escritos lógicos posteriores. ${ }^{40}$ Sin embargo, la DCN impone diversas dificultades, las que tienen su raíz en el modo fluctuante en que ella se articula, principalmente, con los conceptos de posibilidad e imposibilidad, por una parte, y, por otra, ser y no-ser.

\section{El ESTATUS DEL CONCEPTO 'NADA': A MEDio CAMINO ENTRE ELIMINATIVISMO Y CONCEBIBILISMO}

\section{Una de las preguntas inmediatas a que conduce la DCN establecida por} Leibniz, es la de la determinación del estatus modal del concepto 'nada'. Aunque, dada la DCN, parezca natural aceptar la reducción - que, por lo demás Leibniz suele

\footnotetext{
${ }^{39}$ En efecto, así concebida, la DCN no es adecuada. La razón de esta inadecuación es una sutil deficiencia técnica. Ésta consiste en que el definiens del definendum 'N' de la DCN describe no solamente las condiciones de individuación de un sujeto proposicional desprovisto de atributos -como el que está definido por la DCN-, sino también las de un atributo que esté, él mismo, desprovisto de todo atributo: tal es el caso, ciertamente, de un atributo primitivo y simple. Del mismo modo en que el sujeto proposicional caracterizado por la DCN, un atributo primitivo y simple -por definición- tampoco tiene atributo alguno: dada esta condición, ya que él es indefinible, ningún atributo puede predicarse de él. Sin embargo, este atributo no corresponde al concepto 'nada'. El definiens de la DCN más bien describe el definendum ' $\mathrm{N}$ ' -que corresponde al concepto 'nada'-, pero no lo define, ya que aquél describe también las condiciones de individuación de un atributo primitivo y simple, que, en vez de corresponder al concepto 'nada' (Nihil), corresponde al concepto 'algo' (Aliquid). Por consiguiente, pese a que sea cierto que todo sujeto ' $\mathrm{N}$ ' que esté desprovisto de todo atributo corresponda al concepto 'nada', es falso que la condición 'no tener atributos' (definiens) sea suficiente para individualizar un sujeto ' $\mathrm{N}$ ' (definendum). En rigor, la DCN no es una relación de equivalencia, condición necesaria para obtener definiciones adecuadas. Sin embargo, recurriendo al index de la edición de la Akademie, hemos podido constatar que, en al menos un escrito, Leibniz proporciona una definición alternativa de la DCN que, precisamente, enmienda esta inadecuación. El pasaje en cuestión es el siguiente: "[Si] 'A' no es 'N', 'B' no es 'N', 'C' no es 'N', y así sucesivamente, [y] asimismo, [si] 'N' no es 'A', 'N' no es 'B', 'N' no es 'C', entonces se dice que 'N' no es nada. A partir de esta definición, se sigue aquello que se dice vulgarmente: la nada no tiene atributos [o que] el no ser no tiene atributos" (De calculo analytico generale (16781679 (?)), A VI, 4-A, 146). A la luz de esta versión alternativa de la DCN, 'N' (definendum) corresponde a un concepto cuya referencia conceptual es 'nada', si, y sólo si, (definiens) 1) ningún concepto es predicado de 'N'y si, conversamente, 2), ningún concepto es el sujeto de 'N'. Dada esta versión alternativa de la DCN, los atributos primitivos y simples no pueden corresponder al definiens de la DCN, ya que no satisfacen la segunda condición. Así, por medio de esta versión alternativa de la DCN -que podemos distinguir como DCN*-, Leibniz proporciona una definición adecuada del concepto 'nada', ya que ésta sí corresponde a una relación de equivalencia. Dicho lo anterior, y teniendo en consideración esta versión alternativa de la DCN expresada por la $\mathrm{DCN}^{*}$, ya que es difícil pensar que un lógico de la talla de Leibniz no se haya percatado de la inadecuación de la DCN, parece más razonable suponer que, si prefirió conservarla en su "versión estándar", fue sólo con un propósito de simplificación en la escritura, supuesta, por lo demás, la operatividad efectiva que la DCN, pese a su deficiencia, asegura.
}

${ }^{40}$ Cf. Definitiones: aliquid, nihil (1679 (?)), A VI, 4-A, 306; Notationes generales (1683-1685 (?)), A VI, 4-A, 551; De ente, existente, aliquo, nihilo et similibus (1683-1685/6 (?)), A VI, 4-A, 570; Definitionem notionum metaphysicarum atque logicarum (1685 (?)), A VI, 4-A, 625; Definitiones. Notiones. Characteres (1687 (?)), A VI, 4-A, 875; Definitiones : aliquid, nihil, non-ens, ens (1688-1689 (?)), A VI, 4-A, 930; Definitiones : terminus vel aliquid, nihil (1688-1689 (?)), A VI, 4-A, 934; Definitiones : aliquid, nihil, impossible, possible (1689 (?)), A VI, 4-A, 939; De modo distinguendi phaenomena realia ab imaginariis (1683-1685/6 (?)) borrador preparatorio, A VI, 4-B, 1498, y; De Mundo praesenti (1683-1688 (?)), A VI, 4-B, 1506. 
establecer- de este concepto al de 'imposible', esta tesis eliminativista de la nada (TEN) es problemática, pues entra en conflicto con una tesis concebibilista del concepto 'nada' (TCN) con la que Leibniz caracteriza alternativamente el mismo concepto. Así pues, al afirmar la concebibilidad de 'nada' y, eo ipso, su posibilidad, TCN, no sólo contraviene TEN, sino que además se opone a la caracterización económica y negativa que del mismo concepto Leibniz da en su juventud. Determinar entre TEN o TCN es el desafío ontológico al que Leibniz debe responder. Precisamente, en medio del laberinto de la nada, Leibniz se encuentra a medio camino de ambas opciones, pues él parece suscribir a dos criterios incompatibles para caracterizar dicho concepto. Examinemos en detalle esta aporía que representa uno de los obstáculos que Leibniz debe sortear para intentar salir del laberinto.

Desde una perspectiva filosófica ortodoxa, Leibniz establece una correlación entre, por una parte, los conceptos 'nada' e 'imposible' y, por otra, entre 'ser' y 'posible'. Indicio de este enfoque eliminativista del concepto 'nada' parece ser el siguiente pasaje (T1):

(T1) El no-ser o imposible [Non-Ens seu impossibile] es aquello cuya definición encierra 'A y no-A', es decir, aquello que implica contradicción. El ser o posible [Ens seu possibile] es aquello cuya resolución a partir de una definición no encierra 'A y no-A', es decir [que no encierra] una contradicción. ${ }^{41}$

Por medio de una correlación contrastiva, Leibniz identifica o analoga respectivamente los conceptos 'no-ser' y 'ser' a los de 'imposible' y 'posible'. ${ }^{42}$ Descrita en tales términos, T1 no permite, sin embargo, inferir directamente que Leibniz esté identificando o reduciendo el concepto 'nada' al de 'imposible' para respaldar TEN: tal conclusión no es válida a menos que se presuponga, a su vez, la identidad entre los conceptos 'nada' y 'no-ser'. Es precisamente en este dilema en donde recae todo el peso de la dificultad: si, en algunos textos, Leibniz afirma esta identidad -reduciendo el concepto 'nada' al de 'imposible' y validando TEN-, en otros, él la niega -validando una interpretación concebibilista de 'nada' expresada por TCN-. Examinemos, en primer lugar, el primer supuesto, a saber, el de la identidad entre 'nada' y 'no-ser'.

En varios de sus escritos lógicos, Leibniz parece suponer que 'nada' y 'noser' son conceptos equivalentes, dado que ambos se definen por la ausencia de

\footnotetext{
${ }^{41}$ Definitiones: aliquid, nihil, non-ens, ens (1688-1689 (?)), A VI, 4-A, 930. Leibniz subraya.

${ }^{42} \mathrm{Cf}$. Generales Inquisitiones de analysi notionum et veritatum (1686), $\S \S 32$ [bis] y 55, A VI, 4-A, 754 et 757 respectivamente; Specima calculi rationalis (1686 (?)), primero y segundo ensayos, A VI, 4-A, 807 y 810; Definitiones. Notiones. Characteres, A VI, 4-A, 875, y; Definitiones : terminus vel aliquid, nihil, A VI, 4-A, 935.
} 
atributos. Tras formular la DCN en una versión diferente $\left(\mathrm{DCN}^{*}\right),{ }^{43}$ Leibniz afirma la identidad entre 'nada' y 'no-ser' en los siguientes términos (T2):

(T2) A partir de esta definición [DCN*], se sigue aquello que se dice vulgarmente: la nada no tiene atributos [o que] el no ser no tiene atributos. ${ }^{44}$

'Nada' y 'no-ser' son, por consiguiente, términos equivalentes: 'nada' y 'no-ser' coinciden, ya que ambos se definen por la ausencia de todo atributo. Por lo tanto, dado que -según TI- 'no-ser' es puramente privativo, y supuesto que - de acuerdo con T2- 'nada' está "determinado" por la misma condición que 'no-ser', 'nada' carece también de toda propiedad. Es la tesis que Leibniz enuncia claramente en el siguiente pasaje (T3):

(T3) El no-ser es aquello que es puramente privativo, es decir, privativo de todo: 'no-Y', 'no-A', 'no-B', 'no-C'y así sucesivamente. Esto corresponde a lo que uno expresa vulgarmente al decir que la nada no tiene ninguna propiedad. ${ }^{45}$

Dada la conjunción entre T1 y T2-T3, 'nada' y 'no-ser' son lógicamente interdefinibles $\mathrm{y}$, por lo tanto, substituibles, dado que ambos responden a la DCN. Tal es la consecuencia que se desprende del siguiente pasaje (T4):

(T4) La nada [Nihil] es aquello a lo que no corresponde sino términos puramente negativos [terminus mère negativus] (...) $\mathrm{Si}$ ' $\mathrm{N}$ ' no es ' $\mathrm{A}$ ', no es ' $\mathrm{B}$ ', no es ' $\mathrm{C}$ ', no es ' $\mathrm{D}$ ', y así sucesivamente (...) y ningún término positivo que pueda servirle de predicado le es atribuido, entonces se dice que ' $N$ ' corresponde a 'Nada' [Nihil]. Ésa es la razón por la que el axioma común según el cual el no-ser no tiene atributos, contiene la nada misma, es decir, la definición del no-Ser [Non entis nulla sunt attributa, continet ipsius Nihili seu non Entis definitionem]. ${ }^{46}$

T2 y T3 implican la interdefinibilidad y substitutividad entre 'nada' y 'no-ser' afirmada en T4. ${ }^{47}$ Supuesta entonces, por una parte, T1 y, por otra, la relación de implicación entre T2-T3 y T4, por medio de una elemental reconstrucción formal basada en el principio salva veritate de Leibniz, es posible derivar la tesis eliminativista del concepto 'nada' (TEN) a partir de las siguientes premisas:

\footnotetext{
${ }^{43}$ Sobre el significado de DCN*, cf. supra, nota 39.

${ }^{44}$ De calculo analytico generale, A VI, 4-A, 146.

${ }^{45}$ Generales Inquisitiones de analysi notionum et veritatum, A VI, 4-A, 740.

${ }^{46}$ Definitionem notionum metaphysicarum atque logicarum, A VI, 4-A, 625.

${ }^{47}$ Sobre la interdefinibilidad y substitutividad como propiedades operatorias de la identidad, cf. Non inelegans specimen demonstrandi in abstractis (1687 (?)), def. 1, A VI, 4-A, 846.
} 
T1: El no ser no tiene atributos y es imposible

T4: No ser $=$ Nada

TEN: La nada no tiene atributos y es imposible (por salva veritate)

Derivable de la conjunción entre T1 y T4, TEN parece, además de metafísica e intuitivamente plausible, lógicamente garantizada: 'no-ser' y 'nada' son términos idénticos. Sin embargo, TEN no es sostenida incondicionalmente por Leibniz: mientras que algunos textos permiten inferir que TEN es verdadera a condición de negar la identidad entre 'no-ser' y 'nada', otros, derechamente, niegan TEN, suscribiendo Leibniz o a una tesis realista de la nada (TRN) o a una tesis concebibilista de la nada (TCN). Concentrémonos, en primer lugar, en aquellos textos que permiten inferir que Leibniz mantiene TEN, aunque a condición de negar la identidad entre 'no-ser' y 'nada' (T1), continuando el mismo esquema analítico de presentación de tesis. ${ }^{48}$

En una pieza del mismo período, Leibniz niega la identidad entre 'no-ser' y 'nada', incluyendo 'no-ser' dentro de los términos que pueden ser pensados. En efecto, (T5):

(T5) La nada [Nihil] es aquello que se opone a 'algo', es decir, a aquello que puede ser pensado [cogitabile] (ya sea que se trate de un Ser [Ens], ya sea de un 'no-Ser' [non-Ens]). ${ }^{49}$

De acuerdo con T5, 'no-ser' no sólo no es equivalente a 'nada', sino que, al ser concebible y, por lo tanto, ser algo, se opone. De este modo, tanto el concepto 'noser' como el concepto 'ser' son, ambos, posibles. En efecto, (T6):

(T6) Posible es o bien afirmativo [como] el Ser [Ens], o bien negativo, como el No-ser $\left[\right.$ Non-Ens]. ${ }^{50}$

Como es manifiesto a partir de $\mathrm{T} 5$ y T6, lo que resulta concebible no es el concepto 'nada', sino el de 'no-ser', distinguiendo Leibniz éste de aquél. Supuestas T5 y T6, TEN no puede ser entonces verdadera si ella supone la identidad entre 'noser' y 'nada': mientras que 'no-ser' es incluido como un término posible dentro de aquellos conceptos que son pensables, 'nada' parece no estarlo. En efecto (T7):

(T7) Entre los primeros [términos más simples] están aquéllos que son Concebibles [Cogitabile] y que pueden ser pensados de manera confusa o distinta

\footnotetext{
${ }^{48}$ TRN y TCN serán abordadas en las dos últimas secciones respectivamente.

${ }^{49}$ Universum corpus pansophicum (1683 (?)), A VI, 4-B, 1168.

${ }^{50}$ Divisio terminorum ac enumeratio attributorum (1683-1685 (?)), A VI, 4-A, 558. Leibniz entiende así, de manera alternativa, el no-ser como un término: "Yo entiendo por 'Término' tanto el Ser como el No-Ser" (Generales Inquisitiones de analysi notionum et veritatum, A VI, 4-A, 744).
} 
si es imposible que encierren contradicciones. Aquello que puede ser pensado de manera distinta se llama posible y que es, o bien un [término] Positivo como el [término] 'Ser', o bien un [término] negativo como el [término] 'no-Ser'. ${ }^{51}$

En consecuencia, dada T7, tanto el concepto positivo de 'ser' como el negativo de 'noser' son posibles, y, por consiguiente, concebibles ${ }^{52}$. Ahora bien, si T7 garantiza por sí sola la concebibilidad de 'no-ser', es en conjunción con T5 que surge la dificultad, puesto que T5 implica la negación de la identidad entre 'no-ser' y 'nada': T5 y T7 -supuesta T6- implican la concebibilidad de 'no-ser' si, y sólo si, 'no-ser' y 'nada' son distintos, consecuencia que contradice TEN a la luz de T1-T4. Por lo tanto, prima facie, T1-T4 (o sea, TEN) y T5-T7 no pueden ser verdaderas simultáneamente. Y es en este punto en donde se añade una dificultad adicional, pues en ciertos escritos, Leibniz negará directa o indirectamente TEN y en dos sentidos distintos: mientras que ciertos escritos del corpus de Leibniz respaldan una tesis concebibilista de la nada (TCN), otros validan una tesis realista de la nada (TRN).

Supuesto este cuadro, tres son las interpretaciones que podrían caracterizar la doctrina ontológica de la nada en Leibniz: una eliminativista (TEN), una concebibilista $(\mathrm{TCN}) \mathrm{y}$, finalmente, una realista (TRN). Nuestra hipótesis es que la lectura que mejor se adosa a la evidencia textual -sin perjuicio de la persistencia de dificultades tal vez irresolubles- es TCN.

Habiendo ya analizado las tesis que respaldan TEN (T1-T4) y aquéllas que suponen la distinción entre 'no-ser' y 'nada' (T5-T7), expliquemos en qué sentido, a la luz del cuadro antinómico "realismo-anti-realismo de la nada", ciertos textos de Leibniz dan cuenta de TRN, tesis interpretativa que puede constituir una primera tentativa para dar solución el nudo gordiano que apresa en el laberinto ontológico a aquel mismo que lo forjó.

\section{Cortando El NUdo GORDiano: la TESis DEL REALISMo (ANTI-PARMENídeO) DE LA NADA (TRN)}

Una de las posibles soluciones para escapar del laberinto consiste en cortar el nudo gordiano, atribuyendo a la nada un carácter real. Esta respuesta representa, ciertamente, una solución anti-parmenídea, pues, sostener que la nada es tan posible o real como el ser implica contestar los fundamentos mismos de la ontología que,

\footnotetext{
${ }^{51}$ Enumeratio terminorum simpliciorum (1680-1684/5 (?)), A VI, 4-A, 388. Las expresiones que enfatizamos -por medio del uso de cursivas- y las mayúsculas son de Leibniz.

${ }^{52}$ Esta interpretación concebibilista del concepto 'no-ser' es evocada por Leibniz en otros manuscritos. Cf. e.g. Divisio terminorum ac enumeratio attributorum (1683-1685 (?)), A VI, 4-A, 558 y De Mundo praesenti (1683-1688 (?)), A VI, 4-B, 1506. Estos pasajes serán objeto de análisis en la última sección. Cf. infra, sección 5 .
} 
desde Parménides, subyace a toda la metafísica occidental hasta la modernidad y de la cual, por cierto, Leibniz pretende y parece ser un fiel representante. Sin embargo, Leibniz no desestima esta interpretación. Aunque la evidencia textual sobre esta posición de Leibniz es escasa, ella es lo suficientemente clara para mostrar que, al menos, él la tiene en consideración.

Entre los numerosos manuscritos de Leibniz, se encuentra un conjunto copioso de documentos en que él anota y comenta las obras de otros autores. Entre tales documentos, figura un extenso y detallado comentario crítico del Leptotatos de Juan Caramuel Lobkowitz (1606-1682). ${ }^{53}$ En uno de sus comentarios, Leibniz analiza críticamente el siguiente argumento de Caramuel que reformulamos de la siguiente forma:

P1- Sea la proposición $p$ "no hay nada"

P2- Si $p$ es verdadera, entonces $p$ puede ser conocida;

P3- Si $p$ puede ser conocida, entonces hay un ser que puede conocer $p$;

P4- El ser que puede conocer $p$ no puede ser otro que Dios;

P5- Por lo tanto, si $p$ es verdadera, entonces Dios existe;

P6- Pero si Dios existe, entonces es falso (e imposible) que nada exista.

P7- Luego, $p$ es falsa.

P8- Por lo tanto, si $p$ es verdadera, entonces $p$ es falsa. ${ }^{54}$

En respuesta al argumento de Caramuel, Leibniz, por medio del análisis de las condiciones de verdad de la proposición $p$ "no hay nada", objeta la suposición de compatibilidad -que en Caramuel toma, de hecho, la forma de una implicaciónentre $p(\mathrm{P} 1)$ y la existencia de Dios (P5), pues - dice Leibniz- si nada existiera $(p)$, entonces Dios mismo no existiría, conclusión que invalida la solución que Caramuel pretende dar a su propio argumento, quien sugiere, en el fondo, que $p$ es un falsificador de $p$. En palabras de Leibniz (T8):

(T8) Hay una objeción difícil [en respuesta al argumento de Caramuel]: la proposición objetiva 'Dios no existe' es posible (...). Lo que puede no ser, no es Dios. Por lo tanto, Dios no existe. Es posible probar la proposición mayor 'Es posible que no haya nada'. Pero, si no hubiese nada, Dios tampoco existiría. Por lo tanto, es posible probar la proposición mayor 'Dios no existe'. La proposición objetiva 'No hay nada' no es imposible, porque de ella no se siguen dos [proposiciones] contradictorias simultáneamente. Quien piense de otro modo, debe probar que [de esta proposición] se siguen dos [proposiciones] contradictorias simultáneamente.

\footnotetext{
${ }^{53}$ Leptotatos latine subtilissimus Dialectum Metaphysicam brevissimam, facillimam, et significantissimam exhibet, Vigevano, 1671.

${ }^{54}$ Agradezco a mi director de tesis, Jean-Pascal Anfray, las precisiones sobre la formulación del argumento de Caramuel que él, pertinente y gentilmente, me ha hecho notar.
} 
Sin embargo, como la proposición 'No hay nada' es negativa, de ninguna premisa que se le añada puede deducirse una [proposición] afirmativa y, por consiguiente, [tampoco es posible deducir] dos [proposiciones] contradictorias, de las cuales una sea afirmativa y la otra, negativa..$^{55}$

En su orden lógico, el razonamiento de Leibniz parte con el análisis de dicto de la proposición $p$ 'no hay nada' para, enseguida, deducir las consecuencias de re que de ella se siguen, siendo la más relevante la de la inexistencia de Dios. Sin embargo, el orden de presentación del razonamiento es invertido: Leibniz comienza enunciando la conclusión, a saber, la proposición 'Dios no existe' es posible. Esta proposición se sigue de la premisa 'es posible que no exista nada', la que, a su vez, responde a las condiciones que el definendum de la DCN satisface: ya que 'nada' es aquello que no tiene ningún término positivo, a partir de la proposición 'no hay nada' no es posible deducir una contradicción, ya que toda contradicción supone términos positivos y negativos incompatibles. ${ }^{56}$ Por lo tanto, ya que (de dicto) la proposición $p$ 'no hay nada' es posible y no se autofalsifica a sí misma, es posible (de re) que no haya nada. En definitiva -y ésta es la objeción a Caramuel- si nada existiera, Dios tampoco existiría.

Más allá de esta manifiesta dificultad teológica, ${ }^{57}$ T8 no implica la atribución de una realidad ontológica positiva a la nada: muy por el contrario, es porque Leibniz parte de la premisa opuesta que puede deducir que podría no haber habido nada, incluyendo en el dominio cuantificacional del cual 'nada' es índice a Dios. ${ }^{58}$ No obstante, la importancia de T8 es que, en su enunciación, Leibniz concluye no sólo que la proposición $p$ 'no hay nada' es posible (de dicto), sino también que podría no haber habido nada (de re). En

\footnotetext{
${ }^{55}$ Aus und zu Caramuel de Lobkowitz, Leptotatos (1689 (?)), A VI, 4-B, 1338.
}

56 "Todo lo que no implica contradicción es posible" (Essais de Théodicée I, §173, GP VI, 217). Así, supuesta la posibilidad de la nada, la DCN sería entonces una definición real, ya que "las definiciones reales establecen la posibilidad de las cosas definidas" (Meditationes de cognitione, veritate et ideis (1684), A VI, 4-A, 589). Para un examen de los criterios que definen la posibilidad de los conceptos en la metafísica de Leibniz, cf. e.g. G. Martin 1664, pp. 21-29 et 75-83.

${ }^{57} \mathrm{Y}$ que -a nuestro juicio- están directamente conectadas con la pregunta ‘¿Por qué hay algo más bien que nada?', cuya legitimidad Leibniz defiende en el §7 de los Principes de la nature et de la grâce fondés en raison (cf. GP VI, 602), pero que es negada implícitamente en De ratione cur haec existant potius quam alia (1689 (?)), texto que, de hecho, data de la época de los comentarios de la obra de Caramuel (cf. A VI, 4-B, 1634). Para un análisis de estos pasajes y otros afines en el contexto de la teoría de la creación de Leibniz, cf. A. Echavarría (2013).

${ }^{58}$ Así planteada, la cuestión de excluir o incluir a Dios del dominio cuantificacional de la proposición $p$ 'no hay nada' repercute directamente sobre el modo en que la pregunta '¿Por qué hay algo más bien que nada?' debe ser entendida: ¿Debe esta pregunta entenderse como a) ‘¿Por qué hay algo más bien que nada, supuesta la existencia de Dios?' O bien b) ¿Por qué hay algo más bien que nada, e incluso por qué Dios existe?'? La solución a este dilema depende del estatus de la existencia de Dios, cuya determinación exige un análisis preciso de los tres argumentos que Leibniz esgrime para probar su existencia: argumento ontológico (a priori), argumento por la contingencia (a posteriori) y el argumento de las verdades eternas. Para una discusión sobre el problema de la demostración de la existencia de Dios a la luz de estos tres argumentos, cf. J. Jalabert (1960), segunda parte, R. M. Adams (1994), segunda parte y M. Griffin (2013), capítulos 1 y 2. 
otras palabras, si bien Leibniz no atribuye realidad ontológica alguna al concepto 'nada', al juzgar la proposición 'no hay nada' como posible (de dicto) e inferir la posibilidad de la nada (de re), él hace entrar en conflicto dicha proposición con TEN. Al negar TEN, T8 sugiere -lo que podemos llamar- una tesis de realismo débil de la nada (TRdN): la nada, aunque carente de atributos, es posible.

Sin embargo, existe al menos un pasaje en la obra de Leibniz en que éste, más allá de considerar TRdN, atribuye a la nada un estatus ontológico real. Leibniz, en efecto, propone, alternativamente, una tesis de realismo fuerte de la nada (TRfN).

En un breve fragmento de un manuscrito redactado en 1695 y en el que Leibniz aborda el problema del origen del mal -problema que inquieta a Leibniz desde su juventud y que intenta responder definitivamente en sus Essais de Théodicée (1710) - ${ }^{59}$ él, desde una perspectiva anti-parmenídea, atribuye a la nada un estatus real, comparando, de hecho, sus atributos con los de Dios. En esta enigmática pieza, TRdN es afirmada por Leibniz en los siguientes términos (T9):

(T9) La nada [le Néant] es infinita (...) eterna [y] sí tiene atributos comunes con Dios. Ella [la nada] comprende una infinidad de cosas, ya que todas aquellas cosas que no son [toutes celles choses qui ne sont point], están comprendidas en la Nada, y aquéllas que ya no existen, vuelven a la nada [celles qui ne sont plus sont rentrées dans le néant]. ${ }^{60}$

Dos son las observaciones relevantes que amerita T9 en torno al problema que abordamos. La primera es que -como Leibniz afirma- la nada tiene atributos (positivos y, por lo tanto, reales) en común con Dios. Ahora bien, esta "comunión de atributos" puede ser entendida de dos formas: i) o bien hay atributos que, cuantitativamente, inhieren simultáneamente en la nada y Dios, ii) o bien la nada y Dios participan de un mismo atributo cualitativamente. En el fondo, leídas a la luz del principio del praedicatum inest subjecto,${ }^{61}$ una posibilidad es la contraparte

\footnotetext{
${ }^{59}$ Pese a que sobre este problema exista ya una abundante literatura dentro de los estudios leibnizianos, nos limitamos sólo a aconsejar la monografía de P. Rateau (2008), que parece ser hasta ahora el estudio más acabado de la filosofía moral de Leibniz en el contexto de su íntegra evolución genética.

${ }^{60}$ Dialogue effectif sur la liberté de l'homme et sur l'origine du mal (1695), G I, 364.

${ }^{61} \mathrm{La}$ inherencia del predicado en el sujeto es un principio central en la metafísica de Leibniz y que él afirma insistentemente en sus escritos lógicos: "En toda proposición el predicado está en el sujeto, es decir, la noción del predicado está envuelta en la del sujeto" (Calculi universalis investigationes (1679 (?)), A VI, 4-A, 219). Cf también e.g. Elementa calculi (1679), A VI, 4-A, 199; Principia logico-metaphysica (1689 (?)), A VI, 4-B, 1644-1645, y; De natura veritatis, contingentiae et indifferentiae atque de libertate et praedeterminatione (1685-1686), A VI, 4-B, 1515. Sin adentrarnos en este punto, nos limitamos sólo a sugerir la lectura del §47 del Quinto escrito A Clarke (cf. GP VII, 400-402) como un marco de referencia para la discusión de este problema, dada la relevancia de la fórmula que dicho principio ahí adopta y la pertinencia que tiene con respecto al texto que hemos analizado. Para una discusión de los aspectos lógicos y ontológicos envueltos en el principio del praedicatum inest subjecto, cf. e.g. C. D. Broad (1972) y D. Rutherford (1988).
} 
o complemento de la otra, pues cada cual hace de los atributos entidades reales, posición ontológica que, sin embargo, Leibniz rechaza: en virtud del nominalismo al cual éste suscribe, los predicados no son per se reales, sino sólo modos del ser, es decir, del sujeto substancial. No obstante -ésta es nuestra segunda observación-, tal es la posición que Leibniz parece suponer: T9 sugiere que la nada tiene cualidades que la caracterizan (la eternidad y la infinitud) y, además, "hay" objetos que forman parte de ella. Definida en tales términos, la nada, en consecuencia, sería real, dado que, por un lado, contiene atributos positivos, y por otro, constituye una extensión conceptual dentro de la cual caen aquellos objetos que "no son". De manera aún más radical que TRdN, TRfN contesta claramente TEN.

Ahora bien, pese a la claridad con que TRdN en T8 y TRfN en T9 dan cuenta de la adopción de una interpretación realista de la nada, ambas representan, a la luz de la totalidad del corpus leibniziano, más una enigmática excepción que una regla general. Por lo tanto, parece difícil que TRdN y TRfN justifiquen una lectura anti-parmenídea de la doctrina de la nada en Leibniz. En vez de cortar el nudo gordiano, quizás convenga desatarlo.

\section{Desatando El NUdo GORDIANO: LA TESIS DE LA CONCEBIBILIDAD DE LA NADA (TCN)}

De acuerdo con la DCN, el concepto 'nada' no contiene sino atributos negativos. Ahora bien, puesto que para que un concepto sea imposible es necesario que él contenga atributos tanto negativos como positivos incompatibles, el concepto 'nada' no puede ser imposible (TEN). Sin embargo, puesto que, por otra parte, para que un concepto sea real es necesario que él contenga atributos positivos, el concepto 'nada' no puede ser real. Y si bien -como acabamos de ver-, Leibniz parece en ciertos textos sugerir esta interpretación (TRN), la evidencia no parece suficiente. Por consiguiente, en vez de aceptar TEN o TRN, parece pertinente examinar si la interpretación restante, a saber, la tesis de la concebibilidad de la nada (TCN) resulta más satisfactoria. A nuestro juicio, TCN da cuenta, en efecto, de manera más precisa de la doctrina ontológica de la nada en Leibniz: oponiéndose a TEN y TRN, TCN establece que 'nada' es posible, irreal y concebible a la vez. En este sentido, TCN permite explicar, además, en qué sentido y por qué, Leibniz hace del concepto 'nada' un objeto de la ontología.

Varias piezas de distinto tenor parecen respaldar TCN. Situada entre TEN y TRN, TCN establece que 'nada' es un concepto irreal, pero racionalmente posible en la medida en que es concebible. En efecto, además de los pasajes ya citados previamente (T5, T6 y T7), ${ }^{62}$ en otros, Leibniz hace del concepto 'nada' un objeto

\footnotetext{
${ }^{62}$ Cf. supra, sección 3.
} 
racionalmente concebible. Muestra de ello es el siguiente fragmento en el que Leibniz explica (T10):

(T10) [Es necesario considerar] la manera en que es posible llevar a cabo [effectuari] e incluso la manera en que el hombre concibe [intelligat] lo que es el Ser, algo [aliquid], la Nada [Nihil], la Substancia, la Cualidad, el Todo, la Parte, la Acción, la Pasión y los otros modos más generales. ${ }^{63}$

Al igual que las afecciones del ser, la nada es concebible por el entendimiento. Sin embargo, con ello Leibniz quiere decir algo más: la nada es un objeto de la ontología. A partir de una clasificación de categorías transitivas de inclusión, Leibniz identifica el concepto 'nada' como uno de los objetos de la ciencia general. En efecto (T11):

(T11) La ciencia general [Scientia generalis] no es más que la ciencia de todo aquello que es pensable [intellegibilia]. Ella [la ciencia general] no incluye sólo la lógica tal como ella ha sido desarrollada hasta ahora, sino que también [...] la ontología, es decir, la ciencia que tiene por objeto [“el”] algo y la nada [Nihil], el ser y el no ser, la[s] cosa[s] y el modo de la[s] cosa[s], la substancia y el accidente. ${ }^{64}$

Así pues, dado que la nada está comprendida en la ontología, y ésta, a su vez, en la ciencia de todo aquello que es pensable en general, la nada debe poder ser representada, en algún sentido, intelectualmente, esto es, racionalmente concebida.

Ahora bien, la relación de inclusión transitiva que va de la ciencia de todo aquello que es pensable a la nada, es paralela a la relación que -como hemos visto- ${ }^{65}$ Leibniz establece entre todo aquello que es pensable y el no-ser. Como explica Leibniz (T12),

(T12) Todo aquello que es Pensable [Cogitabile] es o bien un Ser [Ens] o bien un No-Ser [Non-Ens]. El ser es aquello de lo cual es posible afirmar algo. El no-ser es aquello que no tiene sino atributos negativos (...). Si 'A' no fuese 'B', ni 'C', ni 'D' y así al infinito, ['A'] correspondería a Nada [Nihil] ${ }^{66}$

Pese a no estar definidos sino por atributos o términos negativos, 'nada' y 'no-ser' son ambos concebibles. Sin embargo, lo que está concedido es aquello mismo que

\footnotetext{
${ }^{63}$ Methodus docendi una popularis altera scientifica perfectior (1683-1685/6 (?)), A VI, 4-A, 582). Las mayúsculas son de Leibniz y las cursivas son mías.

${ }^{64}$ Introductio ad Encyclopaediam arcanam ; sive Initia et Specimena Scientiae Generalis, de instauratione et augmentis scientiarum, deque perficienda mente, et rerum inventionibus, ad publicam felicitatem (1683-1685 (?)), A VI, 4-A, 527. Las cursivas son nuestras.

${ }^{65}$ Cf. supra, sección 3, en particular T5, T6 y T7.

${ }^{66}$ De Mundo praesenti, A VI, 4-B, 1506. Cf. también Enumeratio terminorum simpliciorum, A VI, 4-A, 388.
} 
ha sido, es y puede ser nuevamente puesto en cuestión: ¿En qué sentido la nada y el no-ser pueden ser concebidos si no encierran ninguna realidad? En otros términos: ¿En qué sentido TCN puede ser verdadera, si -de acuerdo con DCN- 'nada' y 'noser' carecen de toda realidad (como hemos supuesto, al afirmar TEN y negar TRN)? El siguiente pasaje, en el que Leibniz distingue "grados" de no-ser-, aporta una pista interesante para dar cuenta del significado del concepto 'nada' en su ontología. En este pasaje, Leibniz declara (T13):

(T13) El no-ser es o bien de Negación [Negationis] o bien de razón [rationis]. La negación pura del No-Ser, tal como la negación de las formas de las cuales un sujeto no es capaz, es puramente privación [purae privatio]. [El no-ser] de razón [es aquél] que no tiene un ser actual ni posible [nullum habet esse nec actuale nec possibile], pero que es imaginado [fingitur], ya sea como una razón razonante [rationis ratiocinantis], es decir, como una quimera [chimaera], o como una razón razonada [[rationis] ratiocionatae $]]$, que tiene un fundamento en la realidad [quod fundamentum in re habet], como el género de una especie. ${ }^{67}$

Según Leibniz, el no-ser puede entenderse no sólo como pura negación o privación, sino también como no-ser de razón. Este último, pese a no tener un ser actual ni posible -precisa- puede, sin embargo, ser imaginado [fingitur], ya sea como no-ser de razón razonante - es decir, cuyo fundamento es puramente racional-, ya sea como no-ser de razón razonada - es decir que, aunque imaginado, sí tiene un fundamento en la realidad. Es este último sentido del no-ser como no-ser de razón razonada el que -nos parece- permite determinar el sentido de 'nada' en la ontología de Leibniz: es porque, sin ser, él mismo, real, el no-ser tiene sin embargo su fundamento en la realidad, que el concepto 'nada' es concebible (imaginado) y es un objeto de la ontología y de la ciencia general. En un acto de trascendencia, la Razón se desborda o doblega a sí misma para concebir aquello mismo que ella no es: no-ser. Dicho de manera aún más radical: es en virtud de un acto de auto-trascendencia que la Razón debe poder concebir la nada como si fuera realmente posible. Sin embargo, como la nada no es real, si ésta es concebible, no es sino en contraste con el ser: ya que la nada no es real, no puede sino ser concebida por una razón razonada que sí es $o$ tiene un ser. Y es porque el Ser tiene una razón-de-ser que la Razón-que es- busca en aquello que ella misma no es su Ser: Dios o la Nada.

\section{OBSERVACIONES FINALES}

Pese a su temprana adhesión al nominalismo, Leibniz da un espacio relevante al concepto 'nada' en su ontología. Tras juzgar que dicho concepto, al estar desprovisto de significado, es inconcebible, Leibniz lo convierte en objeto del

\footnotetext{
${ }^{67}$ Universum corpus pansophicum, A VI, 4-B, 1168. Los subrayados son de Leibniz.
} 
cálculo lógico. El compromiso con el nominalismo es, de ahí en más, abandonado y reemplazado flagrantemente por un escolasticismo que Leibniz no practica sino furtivamente en sus manuscritos personales. Inesperadamente, situado en medio de un laberinto ontológico, Leibniz sistematiza una doctrina de la nada que, sin embargo, no logra plena unicidad. La lógica se revela, en este contexto, como un recurso ambivalente, pues si, por un lado, ella hace posible un tratamiento analítico de las dificultades, por otro, ella sirve de fértil herramienta para cavar en los cimientos más obscuros y profundos de la Razón. El innumerable conjunto de escritos lógicos de cuño aporético que vieron la luz con la pluma de Leibniz revela la búsqueda incesante que éste emprendió para arrojar luz en las tinieblas y encontrar una salida del laberinto. Sin embargo, en vez de aproximarse a hallar el camino de vuelta y sin encontrar, además, una voz con la cual dialogar, él se ve amurallado contra la propia fecundad de su intelecto. La pregunta ‘¿Por qué hay algo más bien que nada?' no es sólo la expresión de un legítimo cuestionamiento teórico: ella es también -y, quizás, sobre todo- la revelación más patente de la desesperación del filósofo que no se conforma con ser 'un petit Dieu'. Pretender responder a tal pregunta es pretender penetrar en la mente del Creador ex nihilo. Como dice Leibniz,

Si Dios es el autor de las cosas, y si Él es soberanamente sabio, uno no podría razonar bien sobre la estructura del universo sin penetrar en la visión de su sabiduría [sans y faire entrer les vues de sa sagesse], del mismo modo en que uno no podría razonar sobre [la estructura] de un edificio sin penetrar en los fines del Arquitecto. ${ }^{68}$

\section{BIBLIOGRAFÍA}

Adams, Robert Merrihew. (1994). Leibniz: Determinist, Theist, Idealist. Oxford: Oxford University Press.

Angelelli, Ignazio. (1965). "Leibniz's Misunderstanding of Nizolius Notion of multitudo", Notre Dame Journal of Formal Logique, vol. 6, 4, pp. 319-322.

Angelelli, Ignazio. (2004). "Leibniz et Nizolius: Réponse à Jean-Baptiste Rauzy", Les cahiers philosophiques de Strasbourg, 18, Deuxième semestre, pp. 215-219.

Antognazza, Maria Rosa. (2009). Leibniz: An intelectual Biography. Cambridge: Cambridge University Press.

\footnotetext{
${ }^{68}$ Réponses aux réflexions qui se trouvent dans le 23 Journal des Savants de cette année touchant les conséquences de quelques endroits de la philosophie de Descartes, GP IV, 339.
} 
Broad, C.D. (1972). "Leibniz's predicate in notion principle and some of its alleged consequences", Leibniz: A collection of critical essays, H. Frankfurt (ed.), pp. 1-18: Anchor Books.

Couturat, Louis. (1961). La Logique de Leibniz, Hildesheim: Georg Olms. (1ª ed. 1901, París: Alcan).

Echavarría, Agustín. (2013). “Creación, nada y participación en Leibniz", Revista Pensamiento, vol. 69, 261, pp. 897-918.

Fichant, Michel. (1998). Science et métaphysique dans Descartes et Leibniz. Paris: Presses Universitaires de France.

Garber, Daniel. (2009). Leibniz: body, substance, monad. Oxford: Oxford University Press. Griffin, Michael. (2013). Leibniz, God and necessity. Cambridge: Cambridge University Press. Jalabert, Jacques. (1960). Le Dieu de Leibniz, Paris: Presses Universitaires de France.

Leibniz, G.W. (1923.-). Sämtliche Schriften und Briefe, Berlin-Brandenburgische Akademie der Wissenschaften et al. (ed.). Darmstadt, Leipzig, Berlín: Akademie-Verlag.

Leibniz, G.W. (1960-1961). Philosophischen Schriften, Gerhardt, C.I. (ed.), 7 vols., Berlín, 1875-1890: Hildesheim.

Leibniz, G.W. (1998). Textes inédits, Grua, G. (ed.), 2 vols., Paris: Presses Universitaires de France.

Look, Brandon. (1999). Leibniz and the 'vinculum substantiale', Studia Leibnitiana, Stuttgart, vol. 30: Franz Steiner Verlag.

Martin, Gottfried (1964). Leibniz: Logic and Metaphysics, translated from German by K.J. Northcott and P.G. Lucas: Manchester University Press.

Mates, Benson. (1979). “The lingua philosophica”, Studia Leibnitiana, 8, pp. 59-66.

Mates, Benson. (1980). "Nominalism and Evander's sword", Studia Leibnitiana Supplementa, 21, pp. 213-225.

Mates, Benson. (1986). The Philosophy of Leibniz, Oxford: Oxford University Press.

Mercer, Christia. (1990). "The Seventeenth-Century Debate between the Moderns and the Aristotelians: Leibniz and Philosophia Reformata", Studia Leibnitiana Supplementa, 27, pp. 18-29.

Mercer, Christia. (2004). "Leibniz and his master: The Correspondence with Jakob Thomasius", Leibniz and his Correspondents, pp. 10-46. P. Lodge (ed.). Cambridge: Cambridge University Press.

Mugnai, Massimo. (1992). Leibniz' Theory of Relations, Studia Leibnitiana Supplementa, 28. Stuttgart: Franz Steiner Verlag.

Rateau, Paul. (2008). La question du mal chez Leibniz: Fondements et élaboration de la Théodicée. Paris: Honoré Champion.

Rauzy, Jean-Baptiste. (2001). La doctrine leibnizienne de la vérité: Aspects logiques et ontologiques, Paris: Vrin. 
Russell, Bertrand. (2005). History of Western Philosophy, London: Routledge. ( $1^{\mathrm{a}} \mathrm{ed}$. George Allen \& Unwin: 1946).

Rutherford, Donald. (1988). "Truth, Predication and Complete Concept of an Individual Substance", Studia Leibnitiana. Sonderhefte, 15, pp. 130-144.

Rutherford, Donald. (1995). Leibniz and the rational order of nature, Cambridge: Cambridge University Press.

Schmitt, Charles. (1971). A critical survey and bibliography of studies on Renaissance Aristotelianism, 1958-1969. Padua: Antenore.

Schmitt, Charles. (1983). Aristotle and the Renaissance. (Martin Classical Lectures 27). Cambridge: Harvard University Press.

Wolff, Francis. (2007). Pourquoi y a-t-il quelque chose plutôt que rien? F. Wolff (ed.). Paris. Éditions Rue D'Ulm: PUF. 